\section{Sub-registro da violência doméstica em adolescentes: a (in)visibilidade na demanda ambulatorial de um serviço de saúde no Recife-PE, Brasil}

\section{Under-reporting of domestic violence among adolescents: the (in)visibility of the demand for outpatients care at a health service in the city of Recife, Brazil}

\begin{abstract}
Objectives: to estimate the under-reporting of domestic violence at a health service for adolescents (aged 10 - 19 years) in Recife, Brazil, comparing prevalences based on medical records and active investigation of cases in the past year and characterizing the victims and risk factors associated with life events.

Methods: a cross-sectional study was carried out with 333 adolescents attended between February and May 2004, using semi-structured questionnaires covering variables relating to the location and type of abuse, age sex, occupation, level of education, religion, drug-use, marital status, household income, and family type. Pearson's chi-square test and logistic regression were used to assess the degree of association, using the Epiinfo 6.04 and SPSS software packages.

Results: of the adolescents interviewed, 41.4\% reported abuse although only $1.8 \%$ had this registered in medical records. Physical and psychological abuse were reported by $66.7 \%$ for each, and sexual violence by $17.7 \%$. A third of the adolescents reported repeated abuse since childhood (20\%). Fathers and step-fathers were the main perpetrators of abuse against both sexes. followed by intimate partners. Factors associate with abuse were: unemployment, low level of education and drug-use $(p<0.05)$

Conclusions: although the prevalence of abuse in the adolescents interviewed was high, it is poorly registered in medical records, suggesting that it is substantially under-reported. The factors identified as being associated with abuse reflect generally unsatisfactory living conditions.
\end{abstract}

Key words Adolescent health, Domestic violence, Epidemiology, Risk factors
Marluce Tavares de Oliveira 1

Maria Luiza Carvalho de Lima 2

Maria Dilma de Alencar Barros 3

Alcieros Martins Paz 4

Andréa Maria Ferreira Barbosa 5

Régia Maria Batista Leite 6

1,3 Faculdade de Ciências Médicas da Universidade de Pernambuco (FCM/UPE). Rua Prof. Arnóbio Marques, 310. Santo Amaro. Recife, PE, Brasil. CEP: 50100-130.

E-mail: tavaresm2002@yahoo.com.br

2 Departamento de Estudos em Saúde Coletiva. Centro de Pesquisas Aggeu agalhães. Fundação Oswaldo Cruz. Recife, PE, Brasil.

4,6 Secretaria Municipal de Saúde da Prefeitura da Cidade do Recife.

5 Universidade de Pernambuco. Campus de Garanhuns. Garanhuns, PE, Brasil.

\section{Resumo}

Objetivos: estimar o sub-registro da violência doméstica em um serviço de atenção a adolescentes (10 a 19 anos) no Recife/PE, Brasil, comparando as prevalências a partir dos prontuários clínicos e de busca ativa de casos no último ano e caracterizar a vítima e fatores de risco associados em eventos ocorridos na vida.

Métodos: corte transversal com 333 adolescentes atendidos entre fevereiro e maio de 2004, com questionário semi-estruturado contendo variáveis sobre situação e tipo de violência; idade, sexo, ocupação, escolaridade, religião, uso de drogas, estado civil, renda familiar e tipo da família. Para avaliar a associação foram utilizados testes de qui-quadrado de Pearson e regressão logística através dos programas Epi-info 6.04 e SPSS.

Resultados: dos adolescentes entrevistados, 41,4\% referiram violência, registrada em apenas $1,8 \%$ dos prontuários. Violência física e a psicológica/moral foi referida por $66,7 \%$, cada e a sexual, por $17,7 \%$. Um terço dos adolescentes referiu agressões repetidas, desde a infância (20\%). Pai e padrasto apareceram como principais agressores nos dois sexos; seguidos pelo parceiro da adolescente. Os fatores associados foram: ausência de ocupação, baixa escolaridade e uso de drogas $(p<0.05)$.

Conclusões: embora a prevalência de violência nos adolescentes entrevistados seja alta, observou-se escasso registro nos prontuários clínicos, denotando sub-registro importante. Os fatores associados identificados refletem condições gerais de vida insatisfatórias.

Palavras-chave Saúde do adolescente, Violência doméstica, Epidemiologia, Fatores de risco 


\section{Introdução}

As causas externas (acidentes e violências) vêm se constituindo relevante problema de saúde em todo mundo. ${ }^{1}$ Entre 1990 e 2000 ocorreram no Brasil 211.938 mortes na faixa etária até 19 anos, por essas causas. ${ }^{2}$ Estudo $^{3}$ realizado em Recife mostrou tendência crescente da mortalidade por causas violentas contra os adolescentes entre 1979 e 1995, mantendo-se em níveis elevados em 2005, com coeficiente de mortalidade por causas externas de 104/100 mil habitantes. 4

De acordo com a Organização Mundial de Saúde (OMS), uma estimativa de 31 mil crianças e jovens menores de 15 anos foram vítimas de homicídios em 2002, principalmente em países não desenvolvidos e em fase de desenvolvimento. 4

Partindo da premissa que as mortes por violência sejam os atos extremos, a magnitude da morbidade por este grupo de causa assumiria patamares mais elevados. Contudo, a subestimação da morbidade por violência se dá pela falta de diagnóstico por parte dos profissionais de saúde que, na sua maioria, encontram-se despreparados para identificar a violência, e pela dificuldade de visibilidade do evento, acarretando ausência de notificação. 5

No caso brasileiro, a violência doméstica, aquela que ocorre no lar, distingue-se da violência intrafamiliar por incluir outros membros do grupo, sem função parental, que convivem esporadicamente, e agregados. 6

O fato de que na maioria das expressões da violência doméstica (maus tratos físicos, abuso sexual, negligência, abuso psicológico) os abusadores são membros da própria família, incluindo os pais, tem levado a se analisar a violência doméstica no modelo de explicação da violência intrafamiliar. ${ }^{7}$ Para alguns autores ${ }^{8,9}$ essas violências são reflexos da violência cultural que se estabelece nas relações de gênero e grupos etários, onde as mulheres, crianças e adolescentes são as principais vítimas e manifestam-se nas relações interpessoais. Esses autores advogam que a cultura da violência é um ciclo que se reproduz e tende a se perpetuar.

Em pesquisa realizada em escolas públicas do município de São Gonçalo/RJ, envolvendo adolescentes, constatou-se que a experiência de sofrer ou impingir abusos físicos é recorrente, evidenciandose que as práticas de bater e apanhar continuam sendo uma forma habitual de resolução de conflitos nas famílias. Do total de 1600 adolescentes entrevistados, $27,7 \%$ informaram ter sofrido abuso físico da mãe e $16,7 \%$, do pai. 10
Alguns fatores são apontados como potencializadores da violência doméstica contra os adolescentes: descontrole emocional, falta de responsabilidade, sentimentos de culpa, uso de drogas pelo adolescente e/ou pelos pais, alcoolismo dos pais, falta de diálogo entre os membros da família, escassez econômica, precárias condições de vida, ausência de rede de apoio à família e consequências da violência estrutural existente na comunidade. ${ }^{11}$

Diante do exposto, cresce a necessidade da ampliação de espaços que possam favorecer a expressão dos casos de violência doméstica contra adolescentes, identificando-os como premissa para que propostas de intervenção possam ser desenvolvidas. Entre eles, destacam-se os serviços que prestam assistência à saúde à população envolvida.

Este estudo teve como objetivos estimar o subregistro da violência doméstica em um serviço de atenção a adolescentes (10 a 19 anos) no Recife/PE, comparando as prevalências a partir dos prontuários clínicos e de busca ativa de casos no último ano e caracterizar a vítima, as principais formas de expressão e fatores de risco associados em eventos ocorridos na vida.

\section{Métodos}

O estudo foi desenvolvido no Centro Integrado de Saúde Amaury de Medeiros / Universidade de Pernambuco (CISAM/UPE). Trata-se de um serviço de saúde público universitário, de referência para a realização do aborto previsto em lei, que tem o Programa de Saúde do Adolescente (PROSAD) implantado.

O desenho de estudo foi de corte transversal, sendo a população de estudo constituída por todos adolescentes, usuários do ambulatório, atendidos no período de fevereiro a maio de 2004. Para definir o número de entrevistas necessárias para a representatividade das variáveis de interesse, a partir da população usuária do serviço, estimada em mil adolescentes, procedeu-se ao cálculo de tamanho amostral. Foi considerada uma prevalência de $14 \%$, referente à frequência da violência sexual obtida no estudo piloto, confiabilidade de $99 \%$, com erro amostral de $1 \%$ e poder de $80 \% .{ }^{12}$ A coleta de dados foi realizada através de prontuários clínicos e entrevistas até que a quantidade estimada para amostra fosse alcançada (333 adolescentes).

A variável dependente foi violência doméstica (VD), investigada para ocorrência do evento alguma vez na vida e no ano anterior. Foi classificada segundo a OMS13 em física, psicológica/moral e sexual. Os conceitos adotados para essas formas de 
violências seguem as do mesmo documento em que: a) violência física significa o uso da força para produzir injúrias, feridas, dor ou incapacidade em outrem; b) violência psicológica ou moral nomeia as agressões verbais ou gestuais com o objetivo de aterrorizar, rejeitar, humilhar a vítima, restringirem a liberdade ou ainda, isolá-la do convívio social; c) violência sexual diz respeito ao ato ou jogo sexual que ocorre nas relações hetero ou homossexuais e visa estimular a vítima ou utilizá-la para obter satisfação sexual e práticas eróticas, pornográficas e sexuais impostas por meio de aliciamento, violência física ou ameaças.

A variável dependente "ter sofrido violência doméstica (VD”, foi coletada a partir de duas fontes de dados:

a) entrevistas com aplicação de questionário estruturado, elaborado pelas pesquisadoras, após teste piloto. O motivo da elaboração do instrumento pelas pesquisadoras deveu-se ao fato de não haver disponibilidade, à época do planejamento e realização do estudo, de instrumentos validados para a população brasileira na faixa etária do estudo, a exemplo da adaptação do Parent-Child Conflict Tactics Scales (CTSPC), por Reichenheim e Moraes. 14 Pelo fato da ocorrência de maus tratos ser de grande impacto, perguntou-se em relação à vida do pesquisado. Apenas neste instrumento de coleta de dados foi investigada a ocorrência do evento alguma vez na vida, como forma de identificar a idade da primeira ocorrência e de exposições repetidas;

b) prontuários agendados para consulta no período de estudo. Nestes, para identificação de caso, utilizou-se hipótese diagnóstica de violência ou referências indiretas de maus tratos em todas as consultas registradas nos últimos 12 meses. Por referência indireta, entende-se o registro de dados de história clínica e/ou encaminhamento a serviços para atendimento de VD: Conselho Tutelar, Gerência de Polícia da Criança e Adolescente (GPCA) e outros.

Como estratégia de qualidade da informação, considerando o escasso preenchimento dos dados referentes às variáveis independentes de estudo nos prontuários clínicos, apenas os dados constantes nos questionários foram considerados como fonte para as mesmas, as quais foram agrupadas em: fatores biológicos (idade, sexo), fatores socioeconômicos (ocupação, escolaridade, religião, estado civil, renda familiar, tipo de família), fatores sócio-culturais (uso de drogas lícitas e/ou ilícitas), relação de parentesco/afinidade com o agressor referido. Para fins de categorização do tipo familiar, utilizou-se a classificação de Prado, 15 a partir da perspectiva dos ciclos de vida:16 a) Família nuclear - constitui-se de duas gerações: os pais (marido e esposa) e os filhos; b) Família extensa - compõe-se da família nuclear e dos membros da família de origem (avós, tios e primos); c) Família uniparental - inclui parentes e amigos, ausência de componentes da família nuclear; d) Família polinuclear - pais que se separaram, recasaram e constituíram novas uniões nucleares, mais de um núcleo familiar; e) Outros arranjos - pais que se casaram uma vez e moram junto com os filhos, ou outros arranjos.

A preparação da equipe, formada por residentes do Programa Multiprofissional em Saúde Coletiva da Faculdade de Ciências Médicas da Universidade de Pernambuco (FCM/UPE), incluiu treinamento dos instrumentos e procedimentos, bem como do primeiro acolhimento dos entrevistados. Negociações prévias com profissionais participantes do serviço (Medicina e Psicologia) foram realizadas, para situações que demandassem necessidade de suporte mais prolongado e/ou específico. Para o desenvolvimento das atividades, realizou-se acompanhamento cotidiano da equipe (supervisões de campo) durante todo o período de coleta de dados, tanto para demandas técnico-operacionais, como para suporte psicológico às entrevistadoras, além de encontros semanais previamente agendados para tal fim.

Os adolescentes foram abordados por ordem de chegada, na sala de espera, sendo considerados critérios de inclusão: estar agendado para atendimento no dia e concordância do adolescente e do seu responsável (se acompanhado) em participar da investigação (com assinatura do Termo de Consentimento Livre e Esclarecido), após os esclarecimentos éticos (Resolução 196/96). As entrevistas foram realizadas em sala reservada, apenas com a presença do adolescente, como forma de garantir a confidencialidade e sigilo das informações prestadas. A impossibilidade em participar nestas condições foi considerada critério de exclusão, pelo fato da presença do responsável poder inibir a livre expressão dos adolescentes entrevistados. A análise de todos os prontuários foi realizada por pesquisadora independente, como forma de minimizar viés de informação, evitando a "intenção de busca" diferenciada de informação, relacionado ao conhecimento prévio da situação de exposição à violência.

Os dados coletados através dos questionários foram codificados e digitados para formação do banco no software Epi6. Foi estimada a prevalência da violência na demanda ambulatorial estudada, segundo sexo e faixa etária. Para descrição do perfil dos adolescentes vítimas de violência doméstica foi 
realizada a distribuição de frequência absoluta e relativa das variáveis dependente e independentes. Para identificar a associação entre variáveis, efetuou-se na primeira etapa o cálculo das odds ratio (OR) brutas, com Intervalo de Confiança de 95\% (IC95\%) e respectivas significâncias em análises univariadas.

Na busca de associações da variável dependente com as demais independentes, 17 os odds ratios foram estimados com relação aos níveis de referência; calculados os IC95\% e os valores de $p$ para os OR (qui-quadrado - $\chi^{2}$ de Pearson), além da razão de verossimilhança. Foram investigados confundimento e interação entre as variáveis que apresentaram associação com a VD. Prosseguiu-se a análise para aquelas variáveis que apresentaram associação menor do que $20 \%$ (valor de $p<0.02$ ), sendo as mesmas introduzidas no modelo multivariado do tipo Forward (regressão logística), com os softwares Epi-info e Statistical Package for the Social Sciences (SPSS). A utilização de modelos logísticos para análise de dados dos estudos de corte transversal tem encontrado espaço na literatura, nas últimas décadas, 18 apresentado-se como modelos estatísticos alternativos quando não se pode estimar diretamente a razão de prevalência (RP) e intervalos de confiança; ajustados para variáveis de confusão.

O projeto foi apreciado pela Comitê de Ética do Centro Integrado de Saúde Amaury de Medeiros, tendo o parecer final aprovado em 05.10.2002.

\section{Resultados}

Encontravam-se agendados para atendimento no PROSAD/CISAM, durante o período de fevereiro a maio de 2004, 436 adolescentes, correspondente ao total de prontuários examinados. Daqueles, 359 compareceram à consulta e foram entrevistados, em todos os dias de funcionamento do ambulatório $\left(2^{\mathrm{a}}\right.$ a $6^{\text {a }}$ feira), segundo ordem de chegada, até serem completados os 333 adolescentes definidos na amostra, o que correspondeu a $92,7 \%$ da demanda. Não ocorreram recusas, mas 26 adolescentes com registro de comparecimento não foram identificadas pelas pesquisadoras e supervisora de campo. As perdas foram isoladas; ao longo dos quatro meses de coleta e em cinco destas situações, foi identificado que apenas a mãe compareceu trazendo resultado de exames, não se conseguindo informações adicionais sobre as 21 remanescentes. Quando se procedeu à investigação do evento violência doméstica nas consultas registradas nos 436 prontuários, nos 12 meses anteriores à consulta atual, foram encontradas anotações referentes à mesma em oito prontuários $(1,8 \%)$, enquanto foi referida por $138(41,4 \%)$ dos adolescentes entrevistados, durante o mesmo período de tempo considerado (Tabela 1).

Quanto à caracterização dos adolescentes vítimas de violência doméstica investigados, optouse por utilizar apenas os dados primários com o instrumento da pesquisa (questionário), em virtude da possibilidade de padronização da forma de obtenção dos dados, o que não seria possível com os dados secundários (prontuários). Observou-se que a maioria era de mulheres solteiras, entre 15 e 19 anos (mais de 90\%), com escolaridade fundamental e algum tipo de ocupação ( $62 \%$ de estudantes e/ou com trabalho remunerado), que viviam em famílias nucleares (pai, mãe e filhos), com uma renda familiar abaixo de três salários mínimos (SM) (84\%; sendo que $42 \%$ referiram menos de um SM). A maioria $(64,2 \%)$ professava alguma religião e $82,3 \%$ referiu não ter vícios (Tabela 2 ).

As manifestações de violência que apresentaram maior prevalência foram a violência física e a psicológica ou moral, sendo $66,7 \%$ em cada uma

\section{Tabela 1}

Distribuição de frequência de adolescentes segundo situação de violência doméstica, sexo e idade. Centro Integrado de Saúde Amaury de Medeiros, Recife, PE, 2004.

\begin{tabular}{|c|c|c|c|c|c|c|c|c|c|}
\hline \multirow{3}{*}{$\begin{array}{l}\text { Situação de } \\
\text { violência } \\
\text { doméstica }\end{array}$} & \multicolumn{4}{|c|}{ Masculino } & \multicolumn{4}{|c|}{ Feminino } & \multirow[t]{3}{*}{ Total } \\
\hline & \multicolumn{2}{|c|}{ 10-14 anos } & \multicolumn{2}{|c|}{ 15-19 anos } & \multicolumn{2}{|c|}{ 10-14 anos } & \multicolumn{2}{|c|}{ 15-19 anos } & \\
\hline & $\mathrm{n}$ & $\%$ & $\mathrm{n}$ & $\%$ & $\mathrm{n}$ & $\%$ & $\mathrm{n}$ & $\%$ & \\
\hline Sim & - & - & 5 & 3,6 & 10 & 7,2 & 123 & 89,2 & 138 \\
\hline Não & 2 & 1,0 & 13 & 6,7 & 19 & 9,7 & 161 & 82,6 & 195 \\
\hline Total & 2 & 0,6 & 18 & 5,4 & 29 & 8,7 & 284 & 85,3 & 333 \\
\hline
\end{tabular}


delas, enquanto a violência sexual foi referida por $17,7 \%$ dos adolescentes. Cerca de $33 \%$ foram agredidos mais de dez vezes e quase $20 \%$ tinham menos de dez anos de idade na primeira ocorrência. E, ainda, $46,4 \%$ permaneciam sofrendo violências. Para todos os tipos de violência doméstica, o pai ou padrasto assumiu posição de destaque como agressor, seguidos pelo esposo da adolescente no abuso sexual (Tabela 3).

A mãe ou madrasta assumiu a terceira posição tanto para a violência moral, como para a física, não sendo referida quando se tratou do abuso sexual. Dos cinco homens que referiram ter sido vítimas de violência, a forma física foi relatada por três, enquanto as formas moral e sexual apresentaram um relato cada (dados não apresentados em tabelas).

No caso da violência física, aqueles que sofreram mais de dez episódios somaram 33,3\% e 46,7\% foram agredidos no ano anterior, ou seja, persistiu a situação de violência. Para a violência moral os percentuais alcançados foram aproximados daqueles encontrados para a violência física $(37,1 \%$ e $52,2 \%$ respectivamente). No caso da violência sexual, $31,8 \%$ sofreram mais de dez agressões e $26,1 \%$ foram agredidas no ano anterior. A diferença para situação de violência no ano anterior presente ou não, no caso da violência sexual foi significativa, $p=0,03$ (dados não apresentados em tabelas).

Tabela 2

Perfil dos adolescentes vítimas de violência doméstica. Centro Integrado de Saúde Amaury de Medeiros, Recife, PE, 2004.

\begin{tabular}{|c|c|c|c|}
\hline Variável & $\mathbf{N}$ & $\%$ & IC95\% \\
\hline \multicolumn{4}{|l|}{ Idade (anos) } \\
\hline $10-14$ & 10 & 7,2 & $3,5-12,9$ \\
\hline $15-19$ & 128 & 92,8 & $87,1-96,5$ \\
\hline \multicolumn{4}{|l|}{ Sexo } \\
\hline Masculino & 5 & 3,6 & $1,2-8,3$ \\
\hline Feminino & 133 & 96,4 & $91,7-98,8$ \\
\hline \multicolumn{4}{|l|}{ Ocupação } \\
\hline Estudante & 69 & 50,0 & $41,4-58,6$ \\
\hline Ocupação remunerada & 17 & 12,3 & $7,3-19,0$ \\
\hline Sem ocupação & 52 & 37,7 & $29,6-46,3$ \\
\hline \multicolumn{4}{|l|}{ Escolaridade } \\
\hline Fundamental & 100 & 72,5 & $64,2-79,7$ \\
\hline Médio & 38 & 27,5 & $20,3-35,7$ \\
\hline \multicolumn{4}{|l|}{ Religião* } \\
\hline Com & 89 & 64,2 & $55,9-72,4$ \\
\hline Sem & 49 & 35,8 & $27,5-44,1$ \\
\hline \multicolumn{4}{|l|}{ Uso de drogas } \\
\hline Sem uso & 98 & 71,0 & $62,7-78,4$ \\
\hline Uso drogas lícitas (álcool, tabaco) & 37 & 26,8 & $19,6-35,0$ \\
\hline Uso drogas ilícitas & 3 & 2,2 & $0,45-6,2$ \\
\hline \multicolumn{4}{|l|}{ Estado civil } \\
\hline Solteiro & 76 & 55,1 & $46,4-63,5$ \\
\hline Casado & 62 & 44,9 & $36,5-53,6$ \\
\hline \multicolumn{4}{|l|}{ Renda familiar (em salário mínimo) } \\
\hline$<1$ & 58 & 42,0 & $33,7-50,7$ \\
\hline 1 a 3 & 58 & 42,0 & $33,7-50,7$ \\
\hline$>3$ & 22 & 16,0 & $10,3-23,1$ \\
\hline \multicolumn{4}{|l|}{ Tipo de Família } \\
\hline Nuclear & 57 & 41,3 & $33,0-50,0$ \\
\hline Uniparenteral & 30 & 21,7 & $15,2-29,6$ \\
\hline Polinuclear & 20 & 14,5 & $9,1-21,5$ \\
\hline Outros arranjos & 31 & 22,4 & $15,8-30,3$ \\
\hline
\end{tabular}

* um caso sem informação. 
Dos 92 adolescentes que sofreram violência física, $42 \%$ não se sentiram agredidos moralmente bem como $34,8 \%$, dos que sofreram agressão sexual. Ainda, dos 23 que sofreram agressão sexual, 10 $(43,5 \%)$ não se sentiram agredidos fisicamente.

Para a violência física e moral, em $13 \%$ os agressores foram adolescentes, os demais agressores estão entre 20 e 70 anos. Os dados mostraram que os agressores sexuais são mais jovens, os adolescentes têm participação de $21,7 \%$ e os demais estão entre 20 e 47 anos (dados não apresentados em tabelas).

$\mathrm{Na}$ análise bivariada, as variáveis que apresentaram associação $(p<0,05)$ com a VD foram: ausência de ocupação, escolaridade fundamental e uso de drogas (Tabela 4). Para análise de confundimento e interação foi investigada a associação entre estas três variáveis, identificando-se associação entre escolaridade e ocupação. A análise estratificada por ocupação, revelou OR de Mantel-Haenszel de 1,72 para a relação entre a VD e escolaridade. A OR sem estratificação foi de 1,85 , o que sugere pequeno confundimento, porém não significativo, o mesmo ocorrendo para a avaliação da interação $(p>0,05)$, não estando estes dados apresentados em tabelas. Uma vez afastado confundimento e interação, procedeu-se à análise multivariada (logística) tipo Forward, incluindo as três variáveis. Nesse modelo final, as variáveis que apresentaram significância estatística $(p<0,05)$ foram: sem ocupação, uso de drogas e escolaridade fundamental, o que sugere que essas variáveis são possíveis fatores de risco para a violência doméstica (Tabela 5).

Tabela 3

Distribuição dos adolescentes conforme tipo ou condições em que ocorrem a violência. Centro Integrado de Saúde Amaury de Medeiros, Recife, PE, 2004.

\begin{tabular}{|c|c|c|c|}
\hline Variável & $\mathbf{N}$ & $\%$ & IC95\% \\
\hline \multicolumn{4}{|l|}{ Tipo da violência* } \\
\hline Física & 92 & 66,7 & $58,1-74,4$ \\
\hline Moral / Psicológica & 92 & 66,7 & $58,1-74,4$ \\
\hline Sexual & 23 & 17,7 & $10,9-23,9$ \\
\hline \multicolumn{4}{|l|}{ Frequência da agressão } \\
\hline 1 a 5 & 67 & 48,6 & $39,9-57,2$ \\
\hline 6 a 10 & 22 & 15,9 & $10,3-23,1$ \\
\hline Mais de 10 & 46 & 33,3 & $25,5-41,8$ \\
\hline Ignorado & 3 & 2,2 & $0,4-6,2$ \\
\hline \multicolumn{4}{|c|}{ Idade na primeira ocorrência (anos) } \\
\hline $0-9$ & 27 & 19,6 & $13,3-27,2$ \\
\hline $10-19$ & 111 & 80,4 & $72,8-86,7$ \\
\hline \multicolumn{4}{|c|}{ Ocorrência no ano anterior à entrevista } \\
\hline Sim & 64 & 46,4 & $37,8-55,1$ \\
\hline Não & 74 & 53,6 & $44,9-62,1$ \\
\hline \multicolumn{4}{|c|}{ Grau de parentesco do agressor** } \\
\hline Pai / padrasto & 66 & 47,8 & $39,2-56,5$ \\
\hline Mãe / madrasta & 35 & 25,4 & $18,3-33,5$ \\
\hline Esposo / Esposa & 47 & 34,0 & $26,2-42,6$ \\
\hline Irmão / irmã & 31 & 22,5 & $15,8-30,3$ \\
\hline Outro parente & 28 & 20,3 & $13,9-28,0$ \\
\hline
\end{tabular}

* Um adolescente pode ter sofrido mais de um tipo de violência; ** Um adolescente pode ter sofrido agressão por parte de mais de uma pessoa. 
Associação entre as variáveis biológicas, socioeconômicas, hábitos e violência doméstica contra adolescentes. Centro Integrado de Saúde Amaury de Medeiros, Recife, PE, 2004.

\begin{tabular}{|c|c|c|c|}
\hline Variável & OR* & IC95\% & $p$ \\
\hline \multicolumn{4}{|l|}{ Idade (anos) } \\
\hline $10-14$ & 1,00 & - & - \\
\hline $15-19$ & 1,54 & $0,70-3,39$ & 0,370 \\
\hline \multicolumn{4}{|l|}{ Sexo } \\
\hline Masculino & 1,00 & - & - \\
\hline Feminino & 2,22 & $0,79-6,25$ & 0,191 \\
\hline \multicolumn{4}{|l|}{ Ocupação } \\
\hline Estudante & 1,00 & - & - \\
\hline Ocupação remunerada & 1,79 & $0,87-3,70$ & \\
\hline Sem ocupação & 2,15 & $1,31-3,51$ & 0,006 \\
\hline \multicolumn{4}{|l|}{ Escolaridade } \\
\hline Média & 1,00 & - & - \\
\hline Fundamental & 1,85 & $1,15-2,29$ & 0,014 \\
\hline \multicolumn{4}{|l|}{ Religião } \\
\hline Com & 1,00 & - & - \\
\hline Sem & 0,85 & $0,53-1,34$ & 0,555 \\
\hline \multicolumn{4}{|l|}{ Uso de drogas } \\
\hline Sem uso & 1,00 & - & - \\
\hline Uso de drogas lícitas & 3,78 & $2,00-7-20$ & $<0,001$ \\
\hline \multicolumn{4}{|l|}{ Estado civil } \\
\hline Solteiro & 1,00 & - & - \\
\hline Casado & 1,20 & $0,75-1,91$ & 0,489 \\
\hline
\end{tabular}

* OR= odds ratio.

\section{Tabela 5}

Modelo final da associação (regressão logística) entre as variáveis biológicas, socioeconômicas, hábitos e violência doméstica contra adolescentes. Centro Integrado de Saúde Amaury de Medeiros, Recife, PE, 2004.

\begin{tabular}{lccc}
\hline Variável & OR & IC95\% & $p$ \\
\hline Ocupação & & & - \\
$\quad$ Estudante & 1,00 & - & 0,204 \\
Com ocupação & 1,62 & $0,76-3,46$ & 0,001 \\
Sem ocupação & 1,93 & $1,15-3,24$ & - \\
Uso de drogas & & - & $<0,001$ \\
Sem uso & 1,00 & $1,94-6,64$ & - \\
$\quad$ Uso de drogas & 2,68 & - & 0,029 \\
Escolaridade & & $1,05-2,82$ & \\
$\quad$ Médio & 1,00 & 1,72 & \\
Fundamental & & & \\
\hline
\end{tabular}

* OR= odds ratio. 


\section{Discussão}

A identificação da violência doméstica, no serviço de saúde analisado, sinaliza para o enorme fosso entre as taxas de prevalência encontradas via questionário e a baixa taxa registrada nos prontuários médicos. Tal achado favorece a emergência da questão da visibilidade/invisibilidade do problema, na dependência da natureza das fontes de dados utilizadas e pode refletir a necessidade de uma qualificação do profissional de saúde para apreender esse fenômeno no dia a dia do seu atendimento. A utilização de instrumento (questionário) que orienta a busca ativa dos casos possibilitou melhor observação dessa visibilidade, denotando um sub-registro considerável, quando comparado com os dados apreendidos nos prontuários preenchidos pelos profissionais de saúde.

Embora aplicado num segmento populacional mais amplo (de mulheres de 15 a 49 anos), estudo conduzido em 2007 em São Paulo, 19 investigando a violência pelo parceiro íntimo e outros agressores, encontrou subnotificação semelhante, denotando a escassa utilidade dos registros clínicos dos serviços de saúde para estudos de prevalência do agravo.

Dois aspectos merecem destaque em relação à população do presente estudo: o primeiro quanto ao predomínio absoluto de mulheres mais velhas (15-19 anos), aproxima-a de populações como as estudadas em serviços de pré-natal,19,20 onde representam parcela importante da demanda. A segunda consideração seria quanto ao processo de individuação e autonomia iniciado com a adolescência. Nessa etapa do ciclo da vida a percepção da dimensão dos direitos não se encontra tão bem estabelecida como na vida adulta, existindo confusão entre o que é direito e o que é dever, ${ }^{21}$ o que pode resultar em avaliações equivocadas na identificação de violências sofridas, atribuindo-as a um "direito" dos pais, resultante de falhas pessoais nos seus 'deveres de filho'. Diante do exposto, acreditamos como lícito levantar a hipótese de que a real magnitude da subnotificação dessas situações seja ainda maior que a encontrada no presente estudo.

O abuso físico foi a expressão mais frequente da violência nos adolescentes estudados, diferentemente de outros estudos 19,20 que encontraram predomínio de abusos psicológicos. Segundo a literatura os abusos físicos são frequentes e tendem a ser repetidos e, quando a vítima é adolescente, crescem as agressões mais graves, o que provoca sérias consequências físicas. Atinge diferentemente meninos e meninas, sendo as mulheres as mais acometidas, 22 assim como nossos dados revelaram.
Embora haja diferenças entre as pesquisas utilizadas para fins de comparação quanto à população envolvida (inclusão de adultos ou apenas adolescentes, como no presente estudo), ao tipo de instrumento utilizado e na maneira de obter as informações, uma das justificativas plausíveis refere-se à baixa autopercepção da violência e suas diferentes formas nesta fase de vida, como comentado anteriormente, na medida em que a percepção dos direitos representa condição sine qua non para a identificação destas formas de agravo, especialmente a violência psicológica por apresentar-se de forma mais sutil e insidiosa.

O fato de o pai ou padrasto assumirem a posição de destaque como agressores, seguidos pelo esposo, respaldam os dados da literatura que apontam para as questões culturais do uso da força física como meio de educar, de resolução de conflitos e como mecanismo de estabelecimento de relações de subordinação e poder que ocorrem no interior das famílias. 19,23 A maioria dos adolescentes pertenciam à família nuclear, o que revela que os pais, ao invés de serem protetores e fonte de afeto e amor, são os primeiros agressores. Consequentemente, o lar, que é também um locus da política, misturada no cotidiano das pessoas, nas discussões dos filhos com os pais e também expressão de afeto, conflitos e contradições, transforma-se em algumas famílias no locus preferencial para violência. 24

Quanto aos outros possíveis fatores de risco apontados pelo estudo (escolaridade baixa, falta de ocupação e uso de drogas), há décadas vem sendo discutido o papel da ausência ou insuficiência de políticas públicas como "pano de fundo" para o estabelecimento de uma série de iniquidades 7 que colaboram para a perpetuação de situações de exclusão social. Assim, por detrás de incapacidades escolares, cristaliza-se a baixa competitividade para o mercado formal e a negação de oportunidades aos jovens das classes populares, levando-os, com certa frequência, às drogas. Dados recentes também vêm abordando a associação da violência com a vivência de situações marginais que, na grande maioria, está ligada ao tráfico de drogas, principalmente nas classes menos favorecidas, como uma saída para o ganho de dinheiro fácil. 2

Nossos dados refletem a mesma abordagem de outros autores, ${ }^{8,9}$ de que a violência doméstica é engendrada na estrutura social que perpassa as questões culturais, e manifesta-se nas relações interpessoais.

Outro aspecto instigante identificado no estudo relaciona-se à participação de adolescentes como perpetradores de violências. Tais achados são 
referidos em artigo, 25 onde a complexa associação de fatores como gênero, idade, temperamento e competência cognitiva, família, inserção social e cultura exercem influência sobre o comportamento de crianças e adolescentes.

No que se refere ao abuso sexual, os estudos mostram que as vítimas são do sexo feminino e os agressores são os pais, o padrasto, namorado, esposo, ou ainda pessoas conhecidas e do relacionamento familiar com a vítima. ${ }^{23}$ Os resultados obtidos em nosso estudo revelaram o mesmo padrão, sugerindo que, quanto mais precoce a idade da vítima, maior a frequência de um padrão de repetição. Assim, a partir de um primeiro episódio de abuso geralmente seguem-se outros, como se houvesse um fio tênue segurando as situações de violência e que uma vez rompido torna-se fácil a repetição.

Estudo 26 mostra que nas famílias onde se desencadeiam episódios de violência "estruturam-se relações familiares disfuncionais, (...) invasivas e/ou sem limites claros, onde climas violentos são compartilhados através da co-participação de cada um dos membros da família na construção da cena violenta". Cria-se então um sistema dinâmico e duradouro, no qual se desenvolvem os papéis de vítima, vitimizador e não-protetor. Este último também participa, conscientemente ou não, da dinâmica estruturante. Pois, "o autor da violência só age como vitimizador na medida em que o parceiro não-protetor autoriza".

Na sua negligência, o pólo não-protetor torna-se um cúmplice da situação, e, em consequência, um vitimizador também. A autora explica como os processos psicológicos que se desencadeiam no ciclo da violência (incluindo a manutenção do segredo), possibilitarão novas invasões/revitimizações do corpo, assim como a reprodução do ciclo violento nas gerações subsequentes.

A ausência de acolhimento dessas vítimas em espaços institucionais ou sociais para que seja rompido o ciclo da violência contribui para a manutenção do silêncio. Por sua vez, as barreiras culturais parecem ser mais difíceis de serem quebradas e denunciadas, provavelmente por constrangimento da vítima de se expor socialmente ou por medo da denúncia poder se constituir em gatilho para novas ameaças. ${ }^{10}$

A natureza repetitiva da violência doméstica indica a importância da detecção precoce e prevenção de problemas futuros dela decorrentes. A busca ativa de casos, através da incorporação rotineira de perguntas na anamnese clínica de usuários de unidades de assistência de saúde, para identificação de situações de violência, tem se mostrado como canal por vezes privilegiado, para emergência da mesma. 22 A atenção ao problema e uma avaliação de saúde mais cuidadosa podem trazer à luz situações de violência subjacentes à queixa referida quando do atendimento. 27

Ainda que o tema da violência contra adolescentes esteja assumindo maior visibilidade nos estudos, pesquisas e publicações, muito ainda resta a investigar para que se tenha uma visão ampliada da questão.

Achados de subnotificação importante, repetição de episódios violentos com início na adolescência, participação de adolescentes como perpetradores de violências, prováveis fatores de risco, além das características sócio-demográficas da população alvo, encontrados no estudo, acompanham os achados de outros estudos descritos na literatura. A personagem típica do nosso estudo: mulher solteira, entre 15 e 19 anos, escolaridade fundamental e algum tipo de ocupação (estudantes e/ou com trabalho remunerado), vivendo em famílias nucleares de baixa renda, com alguma religião e sem vícios declarados.

A diferente distribuição das diversas manifestações da violência e dos agressores referidos identificada nesse estudo, em relação a outros, pode estar relacionada a limitações na comparabilidade dos mesmos, tanto pela diversidade no tipo de instrumento utilizado para obter as informações, como no fato do se incluir população adolescente predominantemente feminina e com pouca representatividade de faixa etária mais jovem (10 a 14 anos).

Embora não tenha havido recusas e os procedimentos de coleta adotados terem garantido a privacidade das informações prestadas, outra provável limitação do estudo diz respeito à exigência legal da assinatura dos pais ou responsáveis do Termo de Consentimento Livre e Esclarecido, para a abordagem de adolescentes em pesquisas. $\mathrm{O}$ fato de os mesmos representarem os principais agressores na maioria dos estudos pode impedir ou dificultar a participação e autonomia de adolescentes referirem maus tratos, revelando magnitude da prevalência do evento menor do que aquela que ocorre na realidade. Esta situação poderia também estar associada à evasão não explicada de 21 adolescentes, o que contribuiria para subestimar os eventos identificados na pesquisa.

Apesar da promulgação do Estatuto da Criança e Adolescente em 1990 e da portaria ministerial de 2001 sobre a Política Nacional de Redução da Morbimortalidade por Acidentes e Violências, que determinam que os profissionais de saúde não 
podem se eximir de sua responsabilidade para identificar, diagnosticar, notificar, prevenir e atuar, esses mecanismos são insuficientes para abordagem do problema. Além disso, observa-se despreparo dos profissionais de saúde que lidam com a problemática, tanto no que diz respeito aos sinais e sintomas sugestivos de abusos, como no manejo de situações identificadas.

A incorporação de busca ativa de casos, aqui realizada, com a utilização de um questionário semiestruturado, apontou para a perspectiva de ampliação das possibilidades de expressão, ponto de partida para aumentar a visibilidade da questão.

A escassa familiaridade com o trabalho partilhado, que inclua diferentes parceiros, de caráter multidisciplinar e intersetorial, dificulta a ativação e manutenção de redes de intervenção e apoio social às situações de violência diagnosticadas.

Assim, para o acolhimento em serviços ambulatoriais de assistência à saúde do adolescente, se faz necessário atuar através de medidas de atenção às vítimas e seus familiares. Recomenda-se que se disponibilize e assegure nos serviços de equipe interdisciplinar com apoio médico, psicológico e social necessário a todos envolvidos no problema, buscando com isto não somente situações de não violência, mas o resgate do seu contraponto, ou seja, a cidadania pela revalorização da vida do ser humano em geral e de cada um em particular, favorecedora de uma saudável convivência.

\section{Referências}

1. WHO (World Health Organization). Preventing child maltreatment: a guide to taking action and generating evidence /World Health Organization and International Society for Prevention of Child Abuse and Neglect. France; 2006. p. 90 .

2. Souza ER, Mello Jorge MH. Impacto da violência na infância e adolescência brasileiras: magnitude da morbimortalidade. In: Lima CA (coord), et al. Violência faz mal à saúde. Brasília: Ministério da Saúde; 2004. p.135-41.

3. Barros MDA, Ximenes R, Lima, MLC. Mortalidade por causas externas em crianças e adolescentes: tendências de 1979 a 1995. Rev Saúde Pública. 2001; 35:142-9.

4. Brasil. Ministério da Saúde/SVS - Sistema de Informações sobre Mortalidade (SIM). Brasília, DF; 2002. [acesso em 7 jun 2009]. Disponível em: http://tabnet.datasus.gov.br/cgi/ idb2007/matriz.htm\#mort

5. Gonçalves HS, Ferreira AL. A notificação da violência intrafamiliar contra crianças e adolescentes por profissionais de saúde. Cad Saúde Pública. 2002; 18: 315-9.

6. Brasil. Ministério da Saúde. Secretaria de Políticas de Saúde. Violência intrafamiliar: orientações para a prática em serviço. Brasília, DF; 2001.

7. Njaine $\mathrm{K}$, Assis $\mathrm{SG}$, Constantino $\mathrm{P}$, org. Impactos da violência na saúde. 2 ed. Rio de Janeiro: Fiocruz; 2009.

8. Assis SG, Avanci JQ. É possível prevenir a violência? Refletindo sobre risco, proteção, prevenção e promoção da saúde. In: Njaine K (org), et al. Impactos da violência na saúde. 2 ed. Rio de Janeiro: Fiocruz; 2009.

9. Brasil. Ministério da Saúde. Sanchéz RN, Minayo MCS Violência contra crianças e adolescentes; questão histórica, social e de saúde. In Lima CA (coord), et al. Violência faz mal à saúde. Brasília, DF; 2004.

10. Assis, SG, Avanci JQ, Santos NC, Malaquias JV, Oliveira RVC. Violência social na adolescência. Rev Panam Saúde Pública. 2004; 16: 43-51.

11. Paludo SS, Koller SH. Toda criança tem família: criança em situação de rua também. Psicol Soc. 2008; 20: 42-52.

12. Lilenfeld AM, Lilenfeld DE. Foundations of epidemiology.
2 ed. New York: Oxford University Press; 1980

13. OMS (Organização Mundial de Saúde). Las formas y los contextos de la violencia. In: Informe mundial sobre la violencia y la salud: resumen. Washington, DC; 2002: 1527.

14. Reichenheim ME, Moraes CL. Portuguese-language crosscultural adaptation of the Parent-Child Conflict Tactics Scales (CTSPC), an instrument used to identify parental violence against children. Cad Saúde Pública. 2003; 19: 1701-12.

15. Prado D. O que é família? São Paulo: Brasiliense; 1981.

16. Pratta EMM, Santos MA. Família e adolescência: a influência do contexto familiar no desenvolvimento psicológico de seus membros. Psicol Estud. 2007; 12: 24756.

17. Kirkwood B, Sterne J. Essential medical estatistics. 2 ed. Oxford, UK: Blackwell publishing; 2003.

18. Coutinho LMS, Scazufca M, Menezes P. Métodos para estimar razão de prevalência em estudos de corte transversal. Rev Saúde Pública. 2008; 42: 992-8.

19. Schraiber LB, D'Oliveira AFPL, Couto MT, Hanada H, Kiss LB, Durand JG, Puccia MI, Andrade MC. Violência contra mulheres entre usuárias de serviços públicos de saúde da Grande São Paulo. Rev Saúde Pública. 2007; 41: 359-67.

20. Schraiber LB, D’Oliveira AF, França-Junior I, Diniz S, Portella AP, Ludermir AB, Valença O, Couto MT. Prevalência da violência contra a mulher por parceiro íntimo em regiões do Brasil. Rev Saúde Pública. 2007; 41: 797-807.

21. Camino C. Concepção das crianças e dos adolescentes sobre direitos humanos, direitos da criança e justiça distributiva. Relatório CNPq; 2004. p. 75-82.

22. Schraiber LB, D'Oliveira AF. Violência contra as mulheres. Interface Comun Saúde Educ. 1999; 3: 11-26.

23. Brasil. Ministério da Saúde. Assis SG, Deslandes SF. Abuso físico em diferentes contextos de socialização infantojuvenil. In: Lima CA (coord), et al. Violência faz mal à saúde. Brasília, DF; 2004. p. 47. 
24. Schraiber LB, D’Oliveira AFPL, Couto MT. Violência e saúde: estudos científicos recentes. Rev Saúde Pública. 2006; 40: 112-20.

25. Garbarino J. Why are adolescents violent?. Ciênc Saúde Coletiva. 2009; 14: 533-8.

26. Brasil. UNICEF. Vecina TCC. Reflexões sobre a construção dos papéis de vítima, vitimizador e não protetor nas situações de violência intrafamiliar. In: Silva HO, Silva JS. Análise da violência contra a criança e o adolescente segundo o ciclo de vida no Brasil: conceitos, dados e proposições. Brasília, DF; 2005. p.18-127.
27. Luna GLM, Ferreira RCF, Vieira JES. Notificação de maus tratos em crianças e adolescentes por profissionais da Equipe Saúde da Família. Ciênc Saúde Coletiva. 2010; 15 : 481-91.

Recebido em 6 de janeiro de 2010

Versão final apresentada em 1 de dezembro de 2010

Aprovado em 3 de janeiro de 2011 